\title{
The effects of DENV serotype competition and co-infection on viral kinetics in Wolbachia-infected and uninfected Aedes aegypti mosquitoes
}

\author{
M. Novelo ${ }^{1,2}$, M. D. Audsley ${ }^{1}$ and E. A. McGraw ${ }^{1,2^{*}}$
}

\begin{abstract}
Background: The Aedes aegypti mosquito is responsible for the transmission of several medically important arthropod-borne viruses, including multiple serotypes of dengue virus (DENV-1,-2,-3, and -4). Competition within the mosquito between DENV serotypes can affect viral infection dynamics, modulating the transmission potential of the pathogen. Vector control remains the main method for limiting dengue fever. The insect endosymbiont Wolbachia pipientis is currently being trialed in field releases globally as a means of biological control because it reduces virus replication inside the mosquito. It is not clear how co-infection between DENV serotypes in the same mosquito might alter the pathogen-blocking phenotype elicited by Wolbachia in Ae. aegypti.

Methods: Five- to 7-day-old female Ae. aegypti from two lines, namely, with (wMel) and without Wolbachia infection (WT), were fed virus-laden blood through an artificial membrane with either a mix of DENV-2 and DENV-3 or the same DENV serotypes singly. Mosquitoes were subsequently incubated inside environmental chambers and collected on the following days post-infection: $3,4,5,7,8,9,11,12$, and 13. Midgut, carcass, and salivary glands were collected from each mosquito at each timepoint and individually analyzed to determine the percentage of DENV infection and viral RNA load via RT-qPCR.
\end{abstract}

Results: We saw that for WT mosquitoes DENV-3 grew to higher viral RNA loads across multiple tissues when coinfected with DENV-2 than when it was in a mono-infection. Additionally, we saw a strong pathogen-blocking phenotype in wMel mosquitoes independent of co-infection status.

Conclusion: In this study, we demonstrated that the wMel mosquito line is capable of blocking DENV serotype co-infection in a systemic way across the mosquito body. Moreover, we showed that for WT mosquitoes, serotype coinfection can affect infection frequency in a tissue- and time-specific manner and that both viruses have the potential of being transmitted simultaneously. Our findings suggest that the long-term efficacy of Wolbachia pathogen blocking is not compromised by arthropod-borne virus co-infection.

Keywords: Dengue, Co-infection, Aedes aegypti, Serotype, Wolbachia, Infection dynamics

*Correspondence: eam7@psu.edu

1 School of Biological Sciences, Monash University, Melbourne, VIC 3800, Australia

Full list of author information is available at the end of the article

\section{Background}

Dengue viruses (DENVs) are medically important arthropod-borne viruses (arboviruses) responsible for up to 300 million cases of dengue fever a year, and they can be caused by any of the four related but antigenically distinct

c) The Author(s) 2021. This article is licensed under a Creative Commons Attribution 4.0 International License, which permits use, sharing, adaptation, distribution and reproduction in any medium or format, as long as you give appropriate credit to the original author(s) and the source, provide a link to the Creative Commons licence, and indicate if changes were made. The images or other third party material in this article are included in the article's Creative Commons licence, unless indicated otherwise in a credit line to the material. If material is not included in the article's Creative Commons licence and your intended use is not permitted by statutory regulation or exceeds the permitted use, you will need to obtain permission directly from the copyright holder. To view a copy of this licence, visit http://creativecommons.org/licenses/by/4.0/. The Creative Commons Public Domain Dedication waiver (http://creativecommons.org/publicdomain/zero/1.0/) applies to the data made available in this article, unless otherwise stated in a credit line to the data. 
DENV serotypes (DENV-1 to DENV-4) [1]. In regions with endemic transmission of all four serotypes of DENV, varying predominance of certain serotypes has been observed between seasons [2]. Differences in the DENV infection kinetics and transmission potential are influenced by the genetic diversity of the four different DENV serotypes. The overall genome sequence-level differences between serotypes are estimated at 20-30\% [3, 4].

Substantial evidence indicates that variation in the DENV genome of serotypes and strains can have epidemiological significance by altering the extrinsic incubation period (EIP) [5-7], defined as the time it takes for the pathogen to be transmitted by the vector [8], and therefore has a powerful effect on the scale and speed of epidemics. DENV-2 strains from the American and Southeast Asian genotypes differ in their EIP lengths, with the Southeast Asian genotypes having shorter EIPs. This shorter EIP was thought, in part, to explain the displacement of the American DENV strains in South America by the Asian lineage [9]. Additionally, different DENV serotypes exhibit various degrees of infectivity across the same mosquito populations [10-14]. Moreover, oral susceptibility to DENV-1 was shown to be up to four times higher than that of DENV-3 in Ae. aegypti from Senegal, with DENV-1 having higher infection and dissemination rates [15]. Finally, systematic analyses of DENV replication kinetics of all four DENV serotypes found significant differences in the infection rate and EIP between serotypes $[13,14]$.

Competition between DENV strains and serotypes can also affect viral population dynamics within the vector, thus modulating the transmission potential $[16,17]$. This happens in nature when mosquitoes take multiple blood meals from several different hosts that are each infected with a different or multiple DENV serotypes [18]. In controlled laboratory experiments, using field-derived mosquito populations, there were no differences in dissemination and transmission rates between DENV-1 and DENV-4 mono-infections in Ae. aegypti, but during co-infection, DENV-4 had a much higher dissemination rate, leading to the exclusive presence of DENV-4 in the saliva for this particular experimental mosquito population [19]. Furthermore, differential replication between DENV-2 and DENV-3 has been shown, with DENV-2 exhibiting a much higher replication efficiency both in vitro and in vivo during co-infection [20]. Additionally, the effect of co-infection with different families of arboviruses on vector competence has just been recently studied. For example, mosquitoes exposed to double or triple infections with chikungunya virus (CHIKV; Togaviridae), Zika virus (ZIKV; Flaviviridae), and DENV (Flaviviridae) were capable of transmitting all pathogens concurrently, without noticeable changes to mosquito infection and dissemination rates [21]. Co-infection studies may shed light on the outcome of competitive processes in field mosquitoes, even if rare, but also importantly allow for direct comparisons of the transmissibility between viruses.

Given the ease of rearing Ae. aegypti in the laboratory, vector competence experiments are an important tool with which to study the effect and interaction between DENV and mosquito genotypes in the transmission potential of the virus. However, one of the main issues with artificial vector competence experiments is that there is too much heterogeneity between experiments that results from environmental variation and its interaction with genetic variation in both the mosquitoes and viruses [22]. Individual vector competence experiments using single DENV serotypes or strains often give varying results in both infection and transmission rates, making pairwise comparisons difficult to interpret. Moreover, limited data are available on co-infections with different serotypes, with some experiments suggesting competitive disadvantage or superinfection interference between DENV serotypes [23]. Additionally, it is not clear how these viral dynamics and interactions may be altered in the presence of Wolbachia infection, which is currently being trialed in global releases as a means of reducing virus transmission to humans [24] and that is known to reduce viral replication in serotype-specific ways [2527]. To the best of our knowledge, only one study has looked at the effects of arboviral co-infection in Wolbachia-infected mosquitoes using ZIKV and DENV [28].

To assess the effect of DENV serotype co-infection on transmissibility and any corresponding interactions with Wolbachia infection, we used two interdependent approaches. First, we challenged two Ae aegypti mosquito lines that were either Wolbachia infected (wMel) or Wolbachia uninfected (WT) in both mono- and coinfection vector competence experiments with DENV-2 and DENV-3. We collected midgut, carcass, and salivary glands at nine time points post-infection. We then used the infection rate and viral RNA load data to assess the effects of competing serotypes and evaluate their performance in individual mosquitoes and between $w \mathrm{Mel}$ and WT lines. Second, we determined whether serotype co-infection altered viral infection dynamics relative to the mono-infected state by comparing viral RNA load and infection rate between the two vector competence experiments.

\section{Methods}

\section{Mosquito lines and rearing}

The mosquito lines used for this work have been described previously $[29,30]$. The $w$ Mel line was collected from the Wolbachia release zone in Cairns, 
Australia, as part of the Eliminate Dengue Program, whereas the WT line, naturally free from Wolbachia, was collected outside the Wolbachia release zone. Both lines were identified morphologically and with genetic markers as well as screened for the presence/absence of pathogens before being used in our study [31]. WT and wMel larvae were fed TetraMin ${ }^{\circledR}$ fish food (Melle, Germany), and adults were maintained on $10 \%$ sucrose. All mosquitoes were reared in a controlled environment at $26{ }^{\circ} \mathrm{C}$, $75 \%$ relative humidity, and a 12-h light/dark cycle.

\section{Virus culture and titration}

The DENV serotypes/strains used for this experiment are listed in Table 1. The virus was propagated in cell culture, as described previously [31]. Briefly, Ae. albopictus C6/36 cells were grown at $26{ }^{\circ} \mathrm{C}$ in RPMI 1640 medium (Invitrogen, Carlsbad, CA) supplemented with $10 \%$ fetal bovine serum (FBS), $1 \times$ GlutaMAX (Invitrogen), and HEPES buffer. Cells were first allowed to form monolayers of around $60-80 \%$ confluence in T-175 flasks (SigmaAldrich, St. Louis, MO), and then they were inoculated with DENV and maintained in RPMI medium supplemented with $2 \%$ FBS. After 7 days post-inoculation, live virus was harvested, titrated via absolute quantification PCR (qPCR) and plaque-forming unit assay (as per below), and adjusted to a final concentration of $\sim 4 \times 10^{5}$ plaque-forming units (PFU)/ml for both serotypes (Table 2) prior to mixing with blood. Live virus was used for all vector competence experiments.

Prior to the above steps, we isolated the viruses at different time points from $\mathrm{C} 6 / 36$ cells and assessed their viral RNA loads by qPCR and plaque assay to select the most appropriate day to harvest virus for the vector competence experiments. These pilot experiments also revealed the relationship between viral RNA load estimates by qPCR and live virus estimates by plaque assay. In general, we saw that higher viral RNA loads correlated with higher plaque assay titers (Additional file 1: Figure 1), with viral RNA loads ranging from $\sim 10$ - to 2000 fold higher than live virus titers, as expected [32, 33]. The average ratio of viral RNA copies relative to $\mathrm{PFU} / \mathrm{ml}$ was larger for DENV-3 (1300-fold) than for DENV-2 (270fold), indicating that RT-qPCR was more sensitive for DENV-3. This effect was consistent across the two tested collection time points.
Table 2 Viral titers

\begin{tabular}{llll}
\hline Serotype & $\begin{array}{l}\text { Undiluted RT-qPCR } \\
\text { titer of infected } \\
\text { supernatant } \\
\text { (copies/ml) }\end{array}$ & $\begin{array}{l}\text { Final plaque assay } \\
\text { titer for the blood } \\
\text { meal (PFU/ml) }\end{array}$ & $\begin{array}{l}\text { Average ratio of } \\
\text { viral RNA copies/ml } \\
\text { relative to PFU/ml }\end{array}$ \\
\hline DENV-2 & $4 \times 10^{7}$ & $\sim 3 \times 10^{5}$ & 277.1 \\
DENV-3 & $8 \times 10^{8}$ & $\sim 4 \times 10^{5}$ & 1382.1 \\
\hline
\end{tabular}

\section{Mosquito infections}

The methods for mosquito oral infections have been described previously $[14,29]$. Briefly, prior to oral DENV infections, 6- to 7-day-old adult female mosquitoes were sorted and starved for $24 \mathrm{~h}$. For mono-infections, a 1:1 mix of virus culture and defibrinated sheep blood was prepared. For co-infections, $1 \mathrm{ml}$ of each DENV serotype was combined, and from that blend, $1 \mathrm{ml}$ was combined with $1 \mathrm{ml}$ of defibrinated sheep blood. Glass feeders with double chambers were covered with pig intestine, and water heated to $37^{\circ} \mathrm{C}$ was circulated in the outer chamber of the feeders. The mosquitoes were allowed to feed for $\sim 2 \mathrm{~h}$. Immediately after blood feeding, mosquitoes were knocked down and sorted on ice. Unfed females were discarded. The remaining mosquitoes were returned to $70-\mathrm{ml}$ plastic cups and maintained on $10 \%$ sucrose. At days post-infection (DPI) 3, 4, 5, 7, 8, 9, 11, 12, and 13, mosquitoes were anesthetized and dissected in sterile phosphate-buffered saline (PBS). We collected midgut, carcass, and salivary glands from individual mosquitoes. For our vector competence experiments, the mosquito carcass was the collection of tissues that remained after dissecting the midgut and salivary glands. We used the carcass as a proxy for viral dissemination from the mosquito midgut. All tissue collections were conducted on live mosquitoes. Individual tissues were collected in 1.5ml microcentrifuge tubes (Sarstedt, Nümbrecht, Germany) containing $200 \mu \mathrm{l}$ of TRIzol reagent (Invitrogen) and 2-mm glass beads. Samples were homogenized and frozen at $-80^{\circ} \mathrm{C}$ until RNA extraction.

\section{DENV absolute quantification via RT-qPCR}

The RT-qPCR mixture contained $2.5 \mu$ of LC480 master $\operatorname{mix}$ (Roche); $4.25 \mu \mathrm{l}$ of PCR-grade water; $0.25 \mu \mathrm{l}$ of $10 \mu \mathrm{M}$ forward $(\mathrm{F})$ primer, reverse $(\mathrm{R})$ primer, and probe $(\mathrm{P})$ specific to each DENV serotype; and $2 \mu$ of RNA. Reactions

Table 1 Dengue serotypes and strains used

\begin{tabular}{llllll}
\hline Serotype & Strain & Passage & GenBank accession number & Place of origin & Collection date \\
\hline DENV-2 & ET300 & 11 & EF440433.1 & East Timor & 2000 \\
DENV-3 & Cairns 2008 & 9 & JN406515.1 & Australia & 2008 \\
\hline
\end{tabular}


were run in the LightCycler 480 instrument (Roche), and the thermal cycling conditions were $95^{\circ} \mathrm{C}$ for $3 \mathrm{~min}, 30$ cycles of $95{ }^{\circ} \mathrm{C}$ for $30 \mathrm{~s}, 50{ }^{\circ} \mathrm{C}$ for $1 \mathrm{~min}$, and $68{ }^{\circ} \mathrm{C}$ for $1 \mathrm{~min}$, finalizing with $68^{\circ} \mathrm{C}$ for $5 \mathrm{~min}$. Standard curves were generated by triplicate on each plate by analyzing 100 to $10^{7}$ copies/reaction of DENV fragment copies with a limit of detection set at 100 copies. DENV genome copies were extrapolated from the standard curve as DENV copies per tissue. The standards for both serotypes were generated using a $\sim 100$-bp conserved region of the NS5 protein of the DENV genome. The detection threshold for both DENV-2 and DENV-3 was set at $35 \mathrm{Ct}$. The primers and probes used for the detection of specific DENV serotypes were as follows:

\section{DENV-2-ET300}

F primer (primer 9,873,681; TCCATACACGCCACA CATGAG).

R primer (primer 98,736,818; GGGATTTCCTCCCAT GATTC).

Probe-FAM (probe 98,084,286; 56-fam/AGGGTG TGGATTCGAGAAAACCCATGG/3BHQ_1).

\section{DENV-3-Cairns08/09}

F primer (primer 98,644,632; TTTCTGCTCCCACCA CTTTC).

R primer (primer 98,451,787; CCATCCYGCTCCCTG AGA).

Probe-LC640 (probe9845178; 5LtC640N/AAGAAA GTTGGTAGTCCCCTGCAGACCTCA/3IAbRQSp).

\section{Statistical analysis}

All statistical analysis used R v3.6.0 (http://www.r-proje ct.org/). For the analysis of the infection frequency, a one-way ANOVA was fitted, and Tukey for contrasts was used for post hoc comparisons. Viral RNA load analysis was carried out using Kruskal-Wallis by rank test for the non-parametric data. All DENV RNA loads were reported on a $\log 10$ scale given the value range.

\section{Results}

In this study, we challenged both WT and $w \mathrm{Mel} A e$. aegypti mosquitoes with two serotypes in the co- and mono-infected states. Specifically, we investigated (1) the relative infection dynamics of two DENV serotypes inside the mosquito by competing them directly, and (2) how individual serotypes behave when they are in a coinfection relative to mono-infection. Mosquitoes were fed blood with DENV-2 and DENV-3, both separately and together. In the case of mono-infections, a 1:1 mix of each DENV serotype and blood was used. For co-infections, the two serotypes were first combined, and from that blend, a 1:1 mix of blood and the two serotypes was used. Overall, the viral titers for both experiments were equivalent. We used RT-qPCR with serotype-specific TaqMan probes to quantify DENV RNA load in midgut, carcass, and salivary gland tissues to assess dissemination and infectiousness at nine time points post-infection.

\section{DENV serotype competition in co-infection}

By competing the serotypes in same mosquitoes, we were able to powerfully compare their performance, controlling some of the substantial variation that can occur across vector competence experiments. Infection rates in the WT mosquito line indicate that DENV-3 was a better competitor than DENV-2 but that the magnitude of this difference changed depending on the tissue and DPI. In the co-infection experiments, we classified the infection rates as uninfected (no viral RNA load detectable for either serotype), only DENV-2 infected (DENV-2/alone), only DENV-3 infected (DENV-3/alone), or co-infected (both serotypes detected). We saw significant variation in the percentage of infected WT mosquitoes between serotypes $(\mathrm{df}=1, F=22.2, P<0.0001)$. In the midgut, most mosquitoes were co-infected at all DPIs (infection rate: $25-100 \%$ ). Mosquitoes were DENV-3/alone at six time points with an infection rate between 10 and 50\%, and DENV-2/alone at DPI 4 and 9 with an infection rate of $\sim 15 \%$ (Fig. 1, midgut).

In the carcass, most WT mosquitoes were either coinfected or DENV-3/alone, but we saw no DENV-2/alone mosquitoes at any time point (Fig. 1, carcass). For the salivary glands, most WT mosquitoes were similarly either co-infected or DENV-3/alone, and there was only one time point at which we observed DENV-2/alone mosquitoes, with an infection frequency of $\sim 10 \%$ at DPI 3 (Fig. 1, salivary glands). For $w$ Mel mosquitoes, no pairwise comparison between serotypes was possible, as there were too few infected mosquitoes to perform statistical analysis, due to the action of Wolbachia-mediated pathogen blocking. Overall, our results indicate that DENV-3 is better at replicating and disseminating in mosquitoes, regardless of tissue or time point, than DENV-2.

We also compared viral RNA loads between serotypes across tissues and DPI. Overall, we saw no significant differences between DENV-2 and DENV-3 (Fig. $2, \chi^{2}=1.66$, $P=0.19, \mathrm{df}=1)$. The viral RNA load for both serotypes in the WT mosquitoes ranged from $\sim 10^{3}$ to $\sim 10^{8}$ in the midgut, $\sim 10^{2}$ to $\sim 10^{7}$ in the carcass, and $\sim 10^{2}$ to $\sim 10^{6}$ $\log 10$ DENV copies per tissue in the salivary glands. In contrast, the viral RNA load in the $w$ Mel mosquitoes was diminished and only observed in 10 time points across all tissues, ranging from $\sim 10^{2}$ to $\sim 10^{5}$ in the midgut, $\sim 10^{2}$ to $\sim 10^{8}$ in the carcass, and $\sim 10^{2} \log 10$ DENV copies per tissue in the salivary glands. Additionally, in the $w \mathrm{Mel}$ mosquitoes, viral RNA loads from both serotypes were 


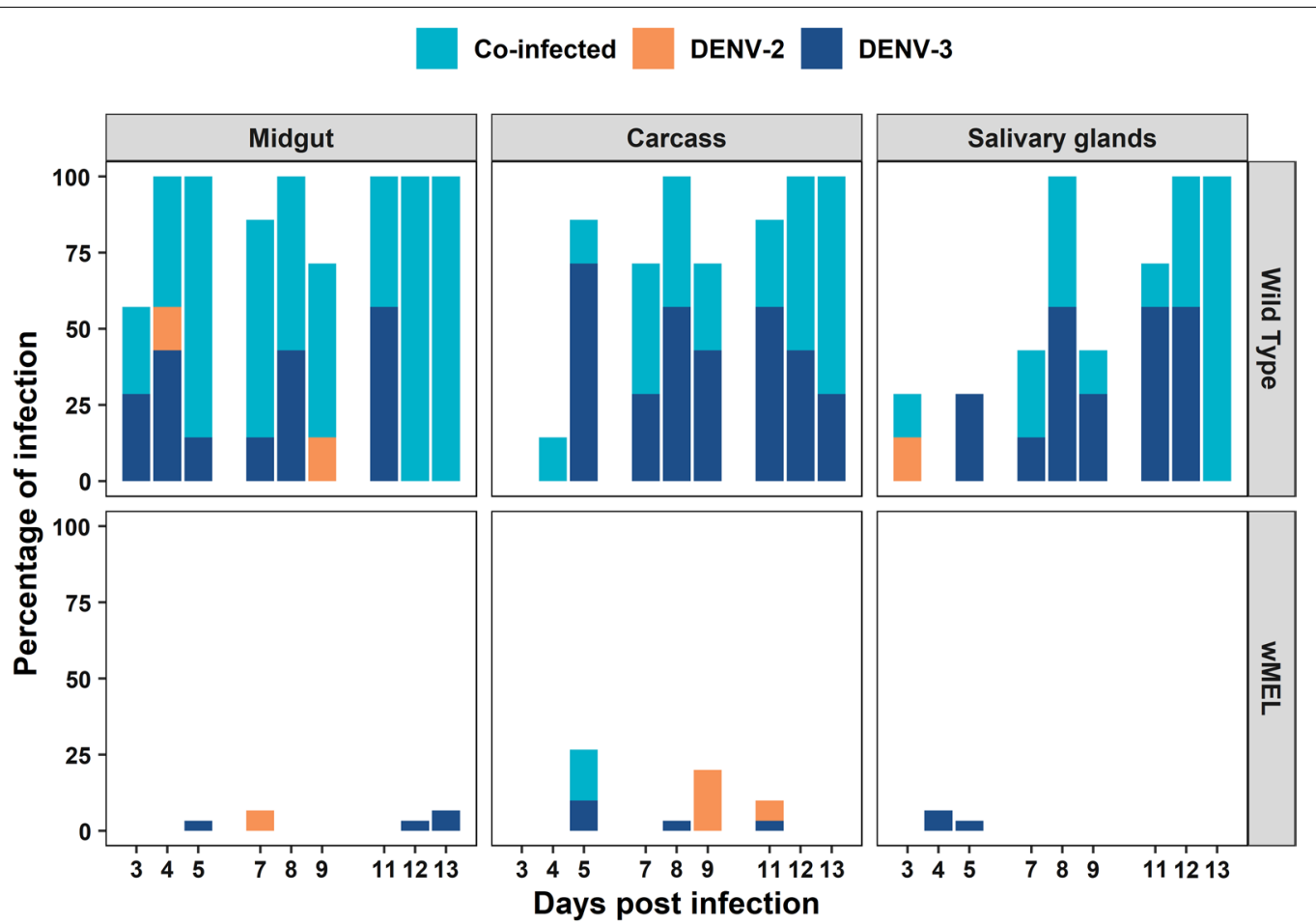

Fig. 1 Mosquito susceptibility to DENV in co-infections by DPI, tissue, and line. Ae. aegypti were orally challenged with DENV-2 (orange) and DENV-3 (navy) simultaneously, whereby mosquitoes were fed a blood meal containing both viruses at $\sim 3 \times 10^{5}$ DENV genome copies $/ \mathrm{ml}$. Mosquito tissues (midgut, carcass, and salivary glands) were collected at nine time points post-infection. DENV RNA load was determined via RT-qPCR using serotype-specific probes. Each bar represents the proportion of mosquitoes positive for either (orange or navy) or both (blue) serotypes for each day post-infection. Total number of mosquitoes screened per day was $n=7$ for the wild-type line and $n=30$ for wMel

observed at only one time point (Fig. 1, wMel, carcass, DPI 5).

Viral RNA loads from both serotypes were expressed as DENV-2/DENV-3 ratio (Fig. 3). Although for all tissues there was a general trend of co-infected samples having higher levels of DENV-3 than DENV-2, they were not significantly different from one another. For the $w \mathrm{Mel}$ mosquitoes, only DPI 5 had sufficient data with which to calculate a ratio due to the action of Wolbachia pathogen blocking; in this case, DENV-3 appeared higher than DENV-2, but with so few data points, statistical comparisons were not possible.

\section{Co-infection alters infection dynamics of DENV serotypes in WT mosquitoes}

After examining the competition dynamics between DENV-2 and DENV-3, we then sought to determine whether or not co-infection altered viral infection dynamics relative to the mono-infected state. For the $w \mathrm{Mel}$ mosquitoes, no pairwise comparisons between mono- and co-infection for each serotype were possible due to the action of Wolbachia-mediated viral blocking. In the WT line, we saw higher infection rates when DENV-3 was in co-infection relative to mono-infection
(Fig. 4) but that the magnitude of this difference varied by DPI and tissue ( $\mathrm{df}=1, F=7.5, P<0.005)$. In the salivary glands, infection was only observed at four time points in the mono-infected state, whereas it was present in eight out of the nine time points in the co-infected mosquitoes (Fig. 4). For viral RNA load, we saw no significant difference between DENV-3 mono- and DENV-3 coinfection (Fig. 5, $\chi^{2}=1.54, P=0.21, \mathrm{df}=1$ ). For DENV-2, there were no differences in either infection frequency (Fig. 4, $\mathrm{df}=1, F=0.97, P=0.33$ ) or viral RNA load (Fig. 5, $\left.X^{2}=0.01, P=0.91, \mathrm{df}=1\right)$ between mono- and co-infection, indicating that co-infection did not alter dynamics.

\section{Tissue-specific differences in viral dynamics}

Overall, for DENV-2, the infection rates were highest in the midgut and declined as the virus moved into the carcass and salivary glands (Fig. 4). DENV-3, in contrast, demonstrated the highest infection rates in the midgut, followed by the salivary glands (Fig. 4). Viral RNA loads for the two serotypes were highest in the midgut and decreased in the carcass and then the salivary glands (Fig. 5, DENV-2; $X^{2}=57.48, P<0.001, \mathrm{df}=2$; DENV-3; $\left.X^{2}=57.48, P<0.001, \mathrm{df}=2\right)$. The carcass was the tissue most likely to have either serotype present despite the 


\section{DENV-2 DENV-3}
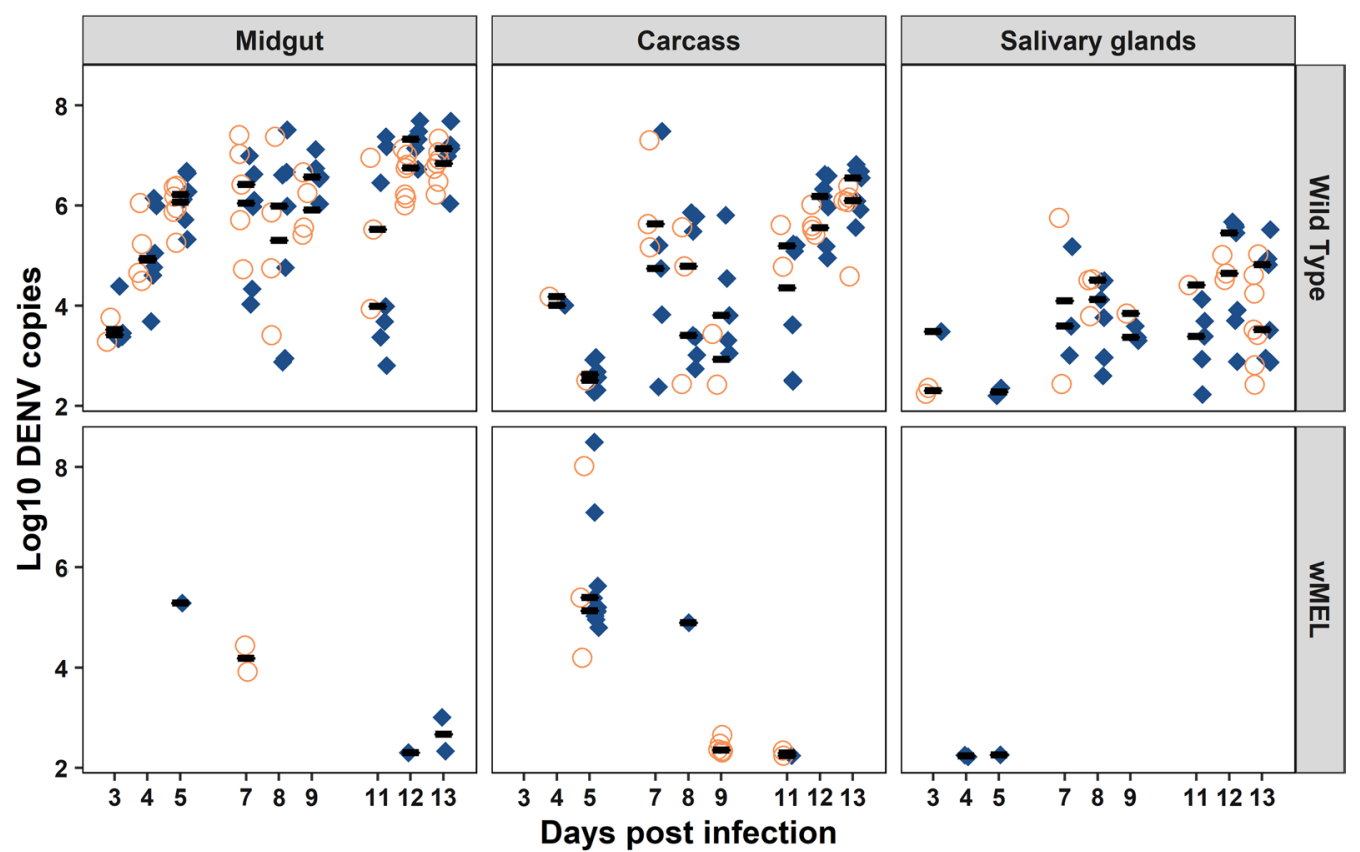

Fig. 2 DENV load in co-infection by DPI, tissue, and mosquito line. Ae. aegypti were orally challenged with DENV-2 (orange circles) and DENV-3 (navy diamonds) simultaneously, and both viruses were fed to mosquitoes at $\sim 3 \times 10^{5} \mathrm{DENV}$ genome copies/ml. Mosquito tissues (midgut, carcass, and salivary glands) were collected at nine days post-infection, and DENV RNA load was determined via RT-qPCR using serotype-specific probes. Mosquitoes with undetectable viral RNA load are not represented in this graph. Black bars represent treatment medians. Each symbol represents a single mosquito sample. Total number of mosquitoes screened per day was $n=7$ for the wild-type line and $n=30$ for $w$ Mel

action of $w$ Mel-mediated pathogen blocking. Dissemination rates of each of the two serotypes in the presence of $w$ Mel were similar across all three tissues (Fig. 1).

\section{Discussion}

We sought to explore the role of DENV serotype coinfection and competition in both Wolbachia-mediated blocking and within-vector infection dynamics. In particular, we examined DENV RNA loads and infection rates in DENV-2/DENV-3 co-infected Ae. aegypti midguts, carcasses, and salivary glands of two mosquito lines with $(w \mathrm{Mel})$ and without Wolbachia infection (WT). We identified that in WT mosquitoes, there was a competitive advantage for DENV-3 when co-infected with DENV-2 across multiple tissues compared to a mono-infection. Additionally, we saw a strong pathogenblocking phenotype in $w \mathrm{Mel}$ mosquitoes independent of co-infection status, tissue, and DPI.

Our data showed that for the WT mosquitoes, DENV serotype co-infection altered the infection frequency of each serotype in a tissue-specific manner, with DENV-3 having a competitive advantage over DENV-2. This advantage was clearer at the dissemination stage of infection once the virus reached the hemocoel from the midgut and ultimately at the transmission level when the virus arrived at the salivary glands. Moreover, the DENV-3 competitive advantage was confirmed when we compared each serotype in co-infected mosquitoes vs mono-infected mosquitoes, and we identified that DENV-3 produced higher infection rates in all tissues when mosquitoes were co-infected. Conversely, DENV-2 infection dynamics did not change significantly when mosquitoes were co-infected compared to monoinfected. Only one other study has looked at the infection dynamics of co-infection with DENV-2 and DENV-3 both in vitro and in vivo [20]; contrary to our findings, they showed an increase in replication efficiency of 1000fold for DENV-2. These contradictory results may be due to the use of different DENV and mosquito genotypes.

When the replication capacities of the two DENV serotypes were assessed in WT mosquitoes, no significant differences in viral RNA load between DENV-2 and DENV-3 in either mono- and co-infection were found. Differences in viral replication rates without affecting the viral RNA load in each tissue have been previously reported [14], in which DENV serotypes that had high growth rates did not necessarily achieve high viral RNA loads or high infection frequencies in experimental 


\section{Wild Type $\because$ wMEL}
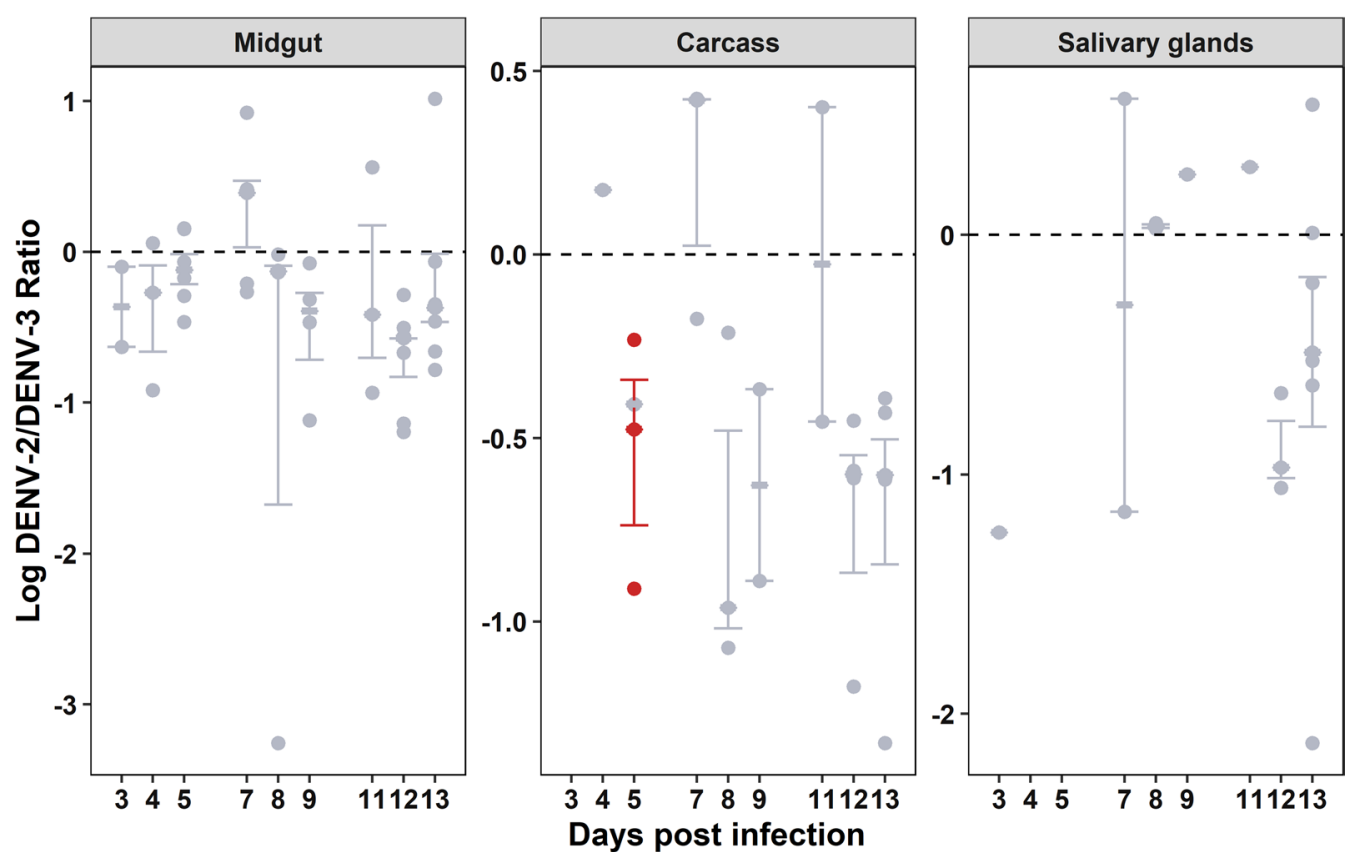

Fig. 3 Co-infection serotype ratio by DPI and tissue. Graphs depict the log 10 DENV-2/DENV-3 ratio for all wild-type (grey) and wMel (red) samples found to be positive for infection with both viruses via RT-qPCR for each tissue. Ratios greater than zero indicate that DENV-2 levels were higher than DENV-3 levels for that sample. Color lines represent mean DENV-2/DENV-3 ratio and the standard error estimate of the mean ratio

mosquito populations. The disconnect between viral RNA load and infection frequency for each virus suggests that stochastic processes are potentially taking place or/ and genotype-by-genotype interactions are affecting the virus infection dynamics. For example, previous research has shown that the strength of the mosquito immune response can be tissue- and serotype-dependent [34-36] and could lead to scenarios in which mosquitoes are more susceptible to dengue infection with a particular serotype (i.e., higher infection frequency) but can also mount a relatively strong immune response in particular tissues (i.e., low viral RNA load).

Arboviral co-infection is not limited to DENV serotypes; although most reported cases are with DENV, co-circulation of DENV with CHIKV and/or ZIKV is increasing around the globe $[37,38]$. This co-circulation represents a major challenge for many national and international public health organizations, particularly because there is little information about the potential clinical and biological consequences of these interactions. Individual case reports of arboviral co-infection in humans suggest enhanced disease severity. Co-infection with ZIKV and CHIKV has been associated with severe meningoencephalitis in a male patient, and co-infection with DENV and CHIKV was linked to severe metrorrhagia in a female patient $[39,40]$. Increased disease severity may occur when both viruses interfere with different parts of the same immune pathways. For example, interferon signaling is a major part of the human antiviral response, and it is mediated by the signal transducer and activator of transcription one and two (STAT-1 and STAT-2) [41]. DENV has been shown to block STAT-1, and CHIKV can potentially interfere with STAT-2 [42], therefore blocking the activation cascade of interferon and potentially increasing disease severity.

The $w$ Mel mosquitoes challenged in DENV serotype co-infection were far less susceptible than the WT line, indicating that the pathogen-blocking phenotype caused by Wolbachia infection is not affected by concomitant DENV serotypes. Additionally, the effect of Wolbachia blocking was seen in all three tissues and was stable across nine time points, from 3 to 13 DPI, encompassing days of the mosquito's lifespan relevant to viral transmission in the field. Pathogen blocking by Wolbachia-infected mosquitoes challenged with co-infecting arboviruses has only been shown once before [43]. Coinfection was performed using DENV/ZIKV challenges but not with multiple DENV serotypes at the same time, and it was limited to three time points and only one mosquito tissue. Although co-circulation of novel emerging 


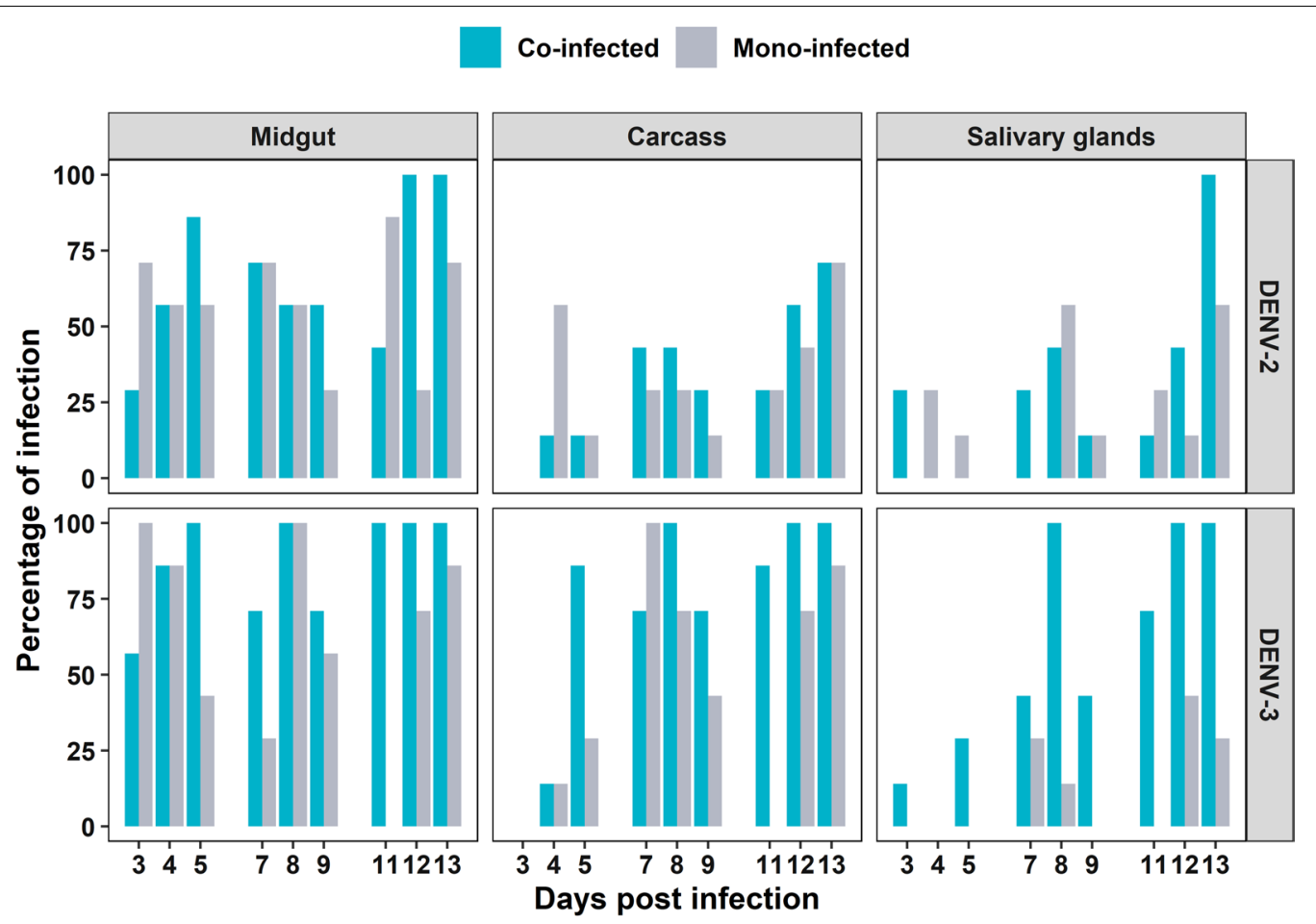

Fig. 4 WT line susceptibility to DENV in co- and mono-infections by DPI, tissue, and serotype. Ae. aegypti mosquitoes were orally challenged with DENV-2 and DENV-3 in both mono- and co-infection experiments. Each bar represents the proportion of mosquitoes positive for either serotype in the mono- (grey) and co-infection (blue) experiments for each day post-infection. Mosquito tissues (midgut, carcass, and salivary glands) were collected at nine days post-infection, and DENV RNA load was determined via RT-qPCR using serotype-specific probes. Sample size $n=7$ per day

arboviruses like ZIKV or CHIKV coupled with DENV has been reported [44-46], most countries where DENV is endemic have reported co-circulation of all four DENV serotypes, resulting in hyper-endemicity for the virus [47, 48]. Specifically, for DENV-2 and DENV-3 infections, one study showed that from 303 human serum samples, up to $21 \%$ were infected with both viruses [49]. This phenomenon has been linked to an increased frequency of severe dengue cases and an overall increase of virulence [50].

Another effect of co-transmission can be epidemiological, where co-infection occurring from a single biting event can significantly increase disease burden. Mathematical modeling using in silico data in Ae. aegypti has shown that co-transmission events can potentially lead to an increased number of cases for both viruses [17]. An additional unanswered question in co-infection in Ae. aegypti is how sequential viral infections might affect pathogen transmission dynamics, a scenario that can also occur in nature $(51,52)$. Whether or not viruses can potentially interact after sequential acquisition by the mosquito has yet to be determined. Last, in our co-infection experiments, we used half as much of each virus but the same overall viral RNA load as mono-infections; however, no statistically significant differences were observed in viral RNA load between mono-infection and co-infection for either serotype. This still begs the question of the potential outcome of infecting the mosquitoes with twice the amount of each serotype and twice the overall amount of virus in co-infection.

\section{Conclusions}

Here, we present the first examination of DENV serotype co-infection and its effect on Wolbachia-mediated pathogen blocking. We demonstrated that the $w \mathrm{Mel}$ mosquito strain is capable of blocking DENV serotype co-infection in a systemic way across the mosquito body. Moreover, we showed that for WT mosquitoes, serotype co-infection can affect infection frequency in a tissue- and timespecific manner and that both viruses have the potential of being transmitted simultaneously. 


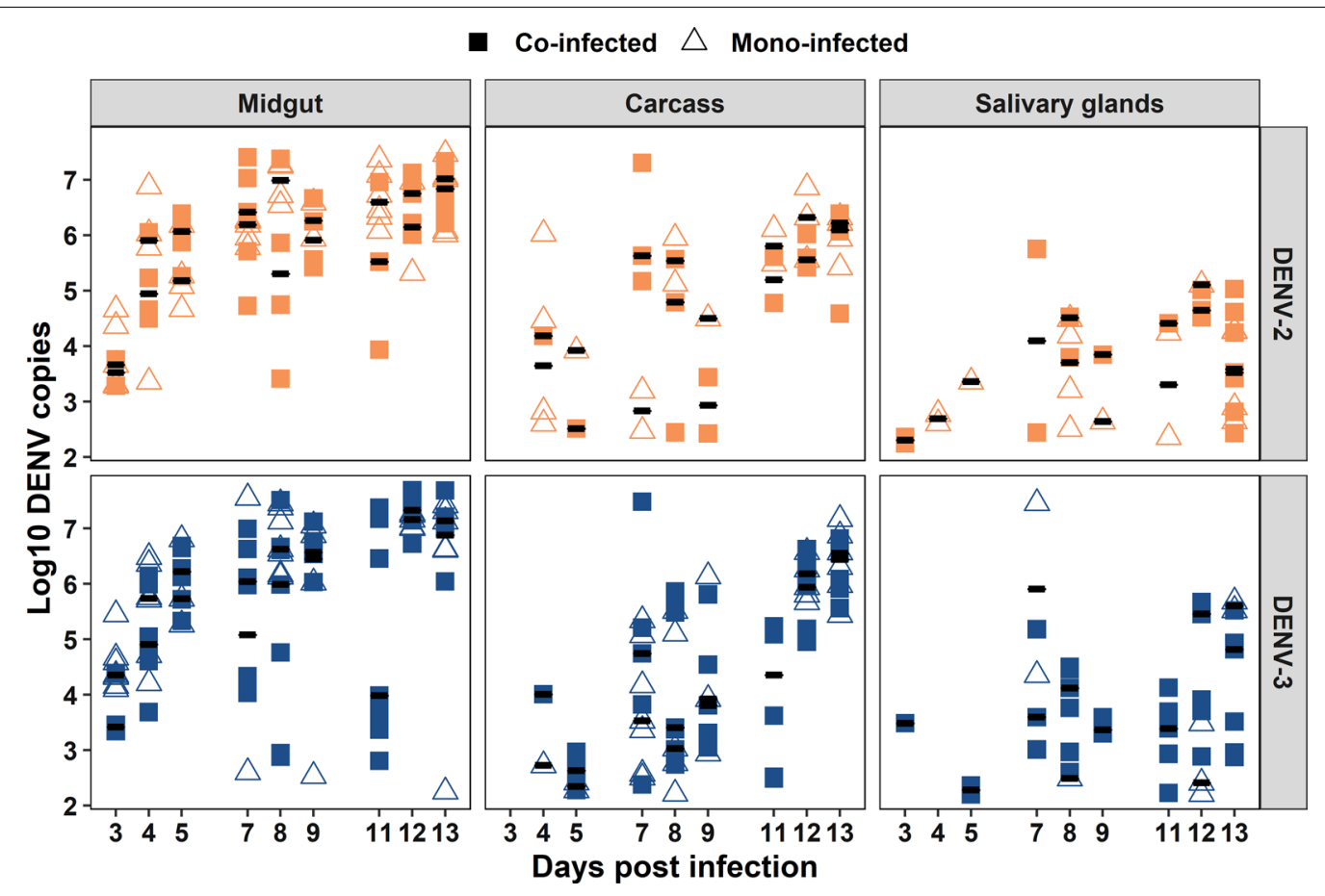

Fig. 5 WT line DENV viral RNA load in co- and mono-infection by DPI, tissue, and serotype. Ae. aegypti mosquitoes were orally challenged with DENV-2 (orange) and DENV-3 (navy) in both co-infection (square) and mono-infection (triangle) experiments, with viruses offered at equivalent titers in both cases. Mosquito tissues (midgut, carcass, and salivary glands) were collected at nine days post-infection, and DENV RNA load was determined via RT-qPCR using serotype-specific probes. Mosquitoes with undetectable viral RNA load are not represented in this graph. Black bars represent treatment medians. Each symbol represents a single mosquito sample. Data were log-transformed. Sample size $n=7$

\section{Supplementary Information}

The online version contains supplementary material available at https://doi. org/10.1186/s13071-021-04816-0.

Additional file 1: Figure S1. Relationship between PFU and viral RNA copies for both DENV-2 and DENV-3. Viruses were harvested from C6/36 cells at DPI 5 and 7 and on the day of the experiment (DPI7-Exp). A) PFU/ $\mathrm{ml}$ for both DENV-2 and DENV-3 for DPI5 (red), DPI7 (blue), and on the day of the experiment DPI7 (black). B) Log10 viral RNA copies/ml for both DENV-2 and DENV-3 for DPI5 (red), DPI7 (blue), and on the day of the experiment DPI7 (black). All assays were done using live virus

\section{Acknowledgements}

We would like to thank E. Kerton, H. Amuzu, G. Terradas, C. Koh, L. Jimenez, S, Ford, and C. Hammer for technical support throughout the experiment. Additionally, we would like to thank Ana Ramírez for sharing and letting us use Ae. aegypti illustrations.

\section{Authors' contributions}

MN participated in the study conception, design, methodology, investigation, formal analysis, and drafted and edited the manuscript. EAM participated in the study conception and design and helped draft and edit the manuscript. MDA participated in the study conception and design and helped edit the manuscript. All authors read and approved the final manuscript.

\section{Funding}

Funded by NHMRC Grant APP1103804 to EAM and NIAID grant 1R01 Al143758 to EAM.
Availability of data and materials

All data for this study can be found at Figshare https://doi.org/10.6084/m9 figshare.14050352.

\section{Declarations}

Ethics approval and consent to participate

Not applicable.

\section{Consent for publication}

Not applicable.

\section{Competing interests}

The authors declare that they have no competing interests.

\section{Author details}

${ }^{1}$ School of Biological Sciences, Monash University, Melbourne, VIC 3800, Australia. ${ }^{2}$ Center for Infectious Disease Dynamics, Department of Entomology, The Huck Institutes of the Life Sciences, The Pennsylvania State University, University Park, PA 16802, USA.

Received: 18 February 2021 Accepted: 29 May 2021

Published online: 09 June 2021

\section{References}

1. Murray NE, Quam MB, Wilder-Smith A. Epidemiology of dengue: past, present and future prospects. Clin Epidemiol. 2013;5:299.

2. Vasilakis N, Weaver SC. The history and evolution of human dengue emergence. Adv Virus Res. 2008;72:1-76. 
3. Rico-Hesse R. Microevolution and virulence of dengue viruses. Adv Virus Res. 2003;59:315-41.

4. Holmes EC, Twiddy SS. The origin, emergence and evolutionary genetics of dengue virus. Infect Genet Evol. 2003;3(1):19-28.

5. Ko H-Y, Salem GM, Chang G-JJ, Chao D-Y. Application of next-generation sequencing to reveal how evolutionary dynamics of viral population shape dengue epidemiology. Front Microbiol. 2020:11:1371.

6. Fontaine A, Lequime S, Moltini-Conclois I, Jiolle D, Leparc-Goffart I, Reiner $\mathrm{RC} \mathrm{Jr}$, et al. Epidemiological significance of dengue virus genetic variation in mosquito infection dynamics. PLoS Pathog. 2018;14(7):e1007187.

7. Lambrechts L, Chevillon C, Albright RG, Thaisomboonsuk B, Richardson $J H$, Jarman RG, et al. Genetic specificity and potential for local adaptation between dengue viruses and mosquito vectors. BMC Evol Biol. 2009;9:160.

8. Macdonald G. The epidemiology and control of malaria. Amen House, Warwick Square, London E.C.4.: Oxford University Press; 1957. xiv;201;11 $\mathrm{pp}$

9. Anderson JR, Rico-Hesse R. Aedes aegypti vectorial capacity is determined by the infecting genotype of dengue virus. Am J Trop Med Hyg. 2006:75:886-92.

10. Weaver SC, Vasilakis N. Molecular evolution of dengue viruses: contributions of phylogenetics to understanding the history and epidemiology of the preeminent arboviral disease. Infect Genet Evol. 2009:9(4):523-40.

11. Gubler DJ, Nalim S, Tan R, Saipan H, Sulianti SJ. Variation in susceptibility to oral infection with dengue viruses among geographic strains of Aedes aegypti. Am J Trop Med Hyg. 1979;28(6):1045-52.

12. Carrington CVF, Foster JE, Pybus OG, Bennett SN, Holmes EC. Invasion and maintenance of dengue virus type 2 and type 4 in the Americas. J Virol. 2005;79(23):14680.

13. Ekwudu OM, Marquart L, Webb L, Lowry KS, Devine GJ, Hugo LE, et al. Effect of serotype and strain diversity on dengue virus replication in Australian mosquito vectors. Pathogens. 2020;9(8):668.

14. Novelo M, Hall MD, Pak D, Young PR, Holmes EC, McGraw EA. Intra-host growth kinetics of dengue virus in the mosquito Aedes aegypti. PLoS Pathog. 2019;15(12):e1008218.

15. Gaye A, Faye O, Diagne CT, Faye O, Diallo D, Weaver SC, et al. Oral susceptibility of Aedes aegypti (Diptera: Culicidae) from Senegal for dengue serotypes 1 and 3 viruses. Trop Med Int Health. 2014;19(11):1355-9.

16. Lambrechts L, Fansiri T, Pongsiri A, Thaisomboonsuk B, Klungthong $\mathrm{C}$, Richardson JH, et al. Dengue-1 virus clade replacement in Thailand associated with enhanced mosquito transmission. J Virol. 2012;86(3):1853-61.

17. Vogels CBF, Rückert C, Cavany SM, Perkins TA, Ebel GD, Grubaugh ND. Arbovirus coinfection and co-transmission: a neglected public health concern? PLoS Biol. 2019;17(1):e3000130

18. Shrivastava S, Tiraki D, Diwan A, Lalwani SK, Modak M, Mishra AC, et al. Co-circulation of all the four dengue virus serotypes and detection of a novel clade of DENV-4 (genotype I) virus in Pune, India during 2016 season. PLOS ONE. 2018;13(2):e0192672.

19. Vazeille M, Gaborit P, Mousson L, Girod R, Failloux AB. Competitive advantage of a dengue 4 virus when co-infecting the mosquito Aedes aegypti with a dengue 1 virus. BMC Infect Dis. 2016;16:318.

20. Quintero-Gil DC, Ospina M, Osorio-Benitez JE, Martinez-Gutierrez M. Differential replication of dengue virus serotypes 2 and 3 in coinfections of C6/36 cells and Aedes aegypti mosquitoes. J Infect Dev Ctries. 2014;8(7):876-84

21. Rückert C, Weger-Lucarelli J, Garcia-Luna SM, Young MC, Byas AD, Murrieta RA, et al. Impact of simultaneous exposure to arboviruses on infection and transmission by Aedes aegypti mosquitoes. Nat Commun. 2017:8:15412

22. Souza-Neto JA, Powell JR, Bonizzoni M. Aedes aegypti vector competence studies: a review. Infect Genet Evol. 2019;67:191-209.

23. Muturi EJ, Buckner E, Bara J. Superinfection interference between dengue-2 and dengue-4 viruses in Aedes aegypti mosquitoes. Trop Med Int Health. 2017:22(4):399-406.

24. Dorigatti I, McCormack C, Nedjati-Gilani G, Ferguson NM. Using Wolbachia for dengue control: insights from modelling. Trends Parasitol. 2018;34(2):102-13

25. Ferguson NM, Kien DTH, Clapham H, Aguas R, Tuan Trung VT, Chau TNB. Wolbachia-mediated interference of dengue virus infection in Aedes aegypti and its projected epidemiological impact on transmission. Sci Transl Med. 2015;7:279ra37.

26. Carrington LB, Tran BCN, Le NTH, Luong TTH, Nguyen TT, Nguyen PT, et al. Field and clinically derived estimates of Wolbachia mediated blocking of dengue virus transmission potential in Aedes aegypti mosquitoes. PNAS. 2018;115(2):361.

27. Bell SM, Katzelnick L, Bedford T. Dengue genetic divergence generates within-serotype antigenic variation, but serotypes dominate evolutionary dynamics. Elife. 2019;8:e42496.

28. Dutra HLC, Rocha Marcele N, Dias FBS, Mansur SB, Caragata EP, Moreira LA. Wolbachia blocks currently circulating Zika virus isolates in Brazilian Aedes aegypti mosquitoes. Cell Host Microbe. 2016;19(6):771-4.

29. Terradas G, Allen SL, Chenoweth SF, McGraw EA. Family level variation in Wolbachia-mediated dengue virus blocking in Aedes aegypti. Parasit Vectors. 2017;10(1):622.

30. Walker T, Johnson PH, Moreira LA, Iturbe-Ormaetxe I, Frentiu FD, McMeniman CJ. The wMel Wolbachia strain blocks dengue and invades caged Aedes aegypti populations. Nature. 2011;476:450-3.

31. Frentiu FD, Robinson J, Young PR, McGraw EA, O'Neill SL. Wolbachiamediated resistance to dengue virus Infection and death at the cellular level. PLoS ONE. 2010;5(10):e13398.

32. Choy MM, Ellis BR, Ellis EM, Gubler DJ. Comparison of the mosquito inoculation technique and quantitative real time polymerase chain reaction to measure dengue virus concentration. Am J Trop Med Hyg. 2013;89(5):1001-5.

33. Richardson J, Molina-Cruz A, Salazar MI, Black WT. Quantitative analysis of dengue-2 virus RNA during the extrinsic incubation period in individual Aedes aegypti. Am J Trop Med Hyg. 2006;74(1):132-41.

34. Cheng G, Liu Y, Wang P, Xiao X. Mosquito defense strategies against viral infection. Trends Parasitol. 2016;32(3):177-86.

35. Taracena ML, Bottino-Rojas V, Talyuli OAC, Walter-Nuno AB, Oliveira $J H M$, Angleró-Rodriguez Yl, et al. Regulation of midgut cell proliferation impacts Aedes aegypti susceptibility to dengue virus. PLoS Negl Trop Dis. 2018;12(5):e0006498.

36. Smartt CT, Shin D, Alto BW. Dengue serotype-specific immune response in Aedes aegypti and Aedes albopictus. Mem Inst Oswaldo Cruz. 2017;112(12):829-37.

37. Bisanzio D, Dzul-Manzanilla F, Gomez-Dantés H, Pavia-Ruz N, Hladish TJ, Lenhart A, et al. Spatio-temporal coherence of dengue, chikungunya and Zika outbreaks in Merida, Mexico. PLoS Negl Trop Dis. 2018;12(3):e0006298

38. Rodriguez-Morales AJ, Villamil-Gómez WE, Franco-Paredes C. The arboviral burden of disease caused by co-circulation and co-infection of dengue, chikungunya and Zika in the Americas. Travel Med Infect Dis. 2016;14(3):177-9.

39. Schilling S, Emmerich P, Günther S, Schmidt-Chanasit J. Dengue and chikungunya virus co-infection in a German traveller. J Clin Virol. 2009;45(2):163-4.

40. Brito CAA, Azevedo F, Cordeiro MT, Marques ETA Jr, Franca RFO. Central and peripheral nervous system involvement caused by Zika and chikungunya coinfection. PLoS Negl Trop Dis. 2017;11(7):e0005583.

41. Murira A, Lamarre A. Type-l interferon responses: from friend to foe in the battle against chronic viral infection. Front Immunol. 2016;7:609.

42. Hollidge BS, Weiss SR, Soldan SS. The role of interferon antagonist, nonstructural proteins in the pathogenesis and emergence of arboviruses. Viruses. 2011;3(6):629-58.

43. Caragata EP, Rocha MN, Pereira TN, Mansur SB, Dutra HLC, Moreira LA. Pathogen blocking in Wolbachia-infected Aedes aegypti is not affected by Zika and dengue virus co-infection. PLoS Negl Trop Dis. 2019;13(5):e0007443-e.

44. Carrillo-Hernández MY, Ruiz-Saenz J, Villamizar LJ, Gómez-Rangel SY, Martínez-Gutierrez M. Co-circulation and simultaneous co-infection of dengue, chikungunya, and Zika viruses in patients with febrile syndrome at the Colombian-Venezuelan border. BMC Infect Dis. 2018;18(1):61.

45. Villamil-Gómez WE, Rodríguez-Morales AJ, Uribe-García AM, GonzálezArismendy E, Castellanos JE, Calvo EP, et al. Zika, dengue, and 
chikungunya co-infection in a pregnant woman from Colombia. Int J Infect Dis. 2016;51:135-8.

46. Dos Santos S, Marinho R, Sanz Duro RL, Santos GL, Hunter J, Da Teles MAR, Brustulin R, et al. Detection of coinfection with chikungunya virus and dengue virus serotype 2 in serum samples of patients in state of Tocantins, Brazil. J Infect Public Health. 2020;13(5):724-9.

47. Villabona-Arenas CJ, De Oliveira JL, Capra CDS, Balarini K, Loureiro $M$, Fonseca CRTP, et al. Detection of four dengue serotypes suggests rise in hyperendemicity in urban centers of Brazil. PLoS Negl Trop Dis. 2014;8(2):e2620-e.

48. Bastos Mde S, Figueiredo RM, Ramasawmy R, Itapirema E, Gimaque JB, Santos LO, et al. Simultaneous circulation of all four dengue serotypes in Manaus, State of Amazonas, Brazil in 2011. Rev Soc Bras Med Trop. 2012:45(3):393-4.

49. Senaratne UTN, Murugananthan K, Sirisena PDNN, Carr JM, Noordeen F. Dengue virus co-infections with multiple serotypes do not result in a different clinical outcome compared to mono-infections. Epidemiol Infect. 2020;148:e119-e.

50. Guzman MG, Harris E. Dengue. Lancet. 2015;385(9966):453-65.

51. Vazeille M, Mousson L, Martin E, Failloux A-B. Orally co-infected Aedes albopictus from la Reunion island, Indian ocean, can deliver both dengue and chikungunya infectious viral particles in their saliva. PLoS Negl Trop Dis. 2010;4(6):e706

52. Nuckols JT, Huang YJ, Higgs S, Miller AL, Pyles RB, Spratt HM, et al. Evaluation of simultaneous transmission of chikungunya virus and dengue virus type 2 in infected Aedes aegypti and Aedes albopictus (Diptera: Culicidae). J Med Entomol. 2015;52(3):447-51.

\section{Publisher's Note}

Springer Nature remains neutral with regard to jurisdictional claims in published maps and institutional affiliations.
Ready to submit your research? Choose BMC and benefit from:

- fast, convenient online submission

- thorough peer review by experienced researchers in your field

- rapid publication on acceptance

- support for research data, including large and complex data types

- gold Open Access which fosters wider collaboration and increased citations

- maximum visibility for your research: over $100 \mathrm{M}$ website views per year

At BMC, research is always in progress.

Learn more biomedcentral.com/submissions 